\title{
Acculturation and the Prevalence of Depression in Older Mexican Americans: Baseline Results of the Sacramento Area Latino Study on Aging
}

\author{
Hector M. González, PhD, * Mary N. Haan, DrPH, * and Ladson Hinton, MD ${ }^{\dagger}$
}

OBJECTIVE: To determine the association between acculturation, immigration, and prevalence of depression in older Mexican Americans.

DESIGN: Cross-sectional analysis from a cohort study.

SETTING: Urban and rural counties of the Central Valley of Northern California.

PARTICIPANTS: One thousand seven hundred and eightynine Latinos recruited from a population-based sample (85\% Mexican Americans) with a mean age of 70.6 (range $60-100$; standard deviation $(\mathrm{SD})=7.13$ ); $58.2 \%$ were women.

MEASUREMENTS: Depressive symptoms were assessed with the Center for Epidemiologic Studies-Depression scale (CES-D). Acculturation was measured with the Acculturation Rating Scale for Mexican Americans-II. Psychosocial, behavioral, and medical histories were also obtained.

RESULTS: The prevalence of depression (CES-D $\geq 16$ ) was $25.4 \%$. Women were at greater risk $(32.0 \%)$ than men $(16.3 \%$; male/female odds ratio $(\mathrm{OR})=2.43,95 \%$ confidence interval $(\mathrm{CI})=1.90-3.09)$. The prevalence of depression was higher among immigrants $(30.4 \%$, OR $=$ $1.70,95 \% \mathrm{CI}=1.36-2.13)$, bicultural participants $(24.2 \%$, $\mathrm{OR}=1.66,95 \% \mathrm{CI}=1.24-2.24)$, and less-acculturated participants $(36.1 \%, \mathrm{OR}=2.95,95 \% \mathrm{CI}=2.22-3.93)$ compared with U.S.-born $(20.5 \%)$ and more-acculturated groups $(16.1 \%)$. When adjustments for education, income, psychosocial, behavioral, and health-problem factors were made, the least-acculturated participants were at significantly higher risk of depression than highly acculturated Mexican Americans $(\mathrm{OR}=1.56,95 \% \mathrm{CI}=1.06-2.31)$.

From the *Department of Epidemiology, School of Public Health, University of Michigan, Ann Arbor, Michigan; and ${ }^{\dagger}$ Department of Psychiatry, School of Medicine, University of California, Davis, Davis, California. Supported by grants from the National Institute on Aging: AG12975 (M. Haan), and Minority Investigator Research Supplement to AG12975 (H. González).

Address correspondence to Hector M. González, PhD, University of Michigan, School of Public Health, Department of Epidemiology, 109 Observatory St., 3007 SPH I, Ann Arbor, MI 48109-2029.
CONCLUSIONS: These findings are consistent with previously reported estimates of a higher prevalence of depression for older Mexican Americans than non-Hispanic Caucasians and African Americans and are the first to report the prevalence and risk of depression for older U.S.born and immigrant Mexican Americans. The high prevalence of depression of the least acculturated group may be related to cultural barriers encountered by immigrants and less-acculturated older Mexican Americans and to poorer health status. J Am Geriatr Soc 49:948-953, 2001.

Key words: depression; Hispanic Americans; Mexican Americans; acculturation; geriatric

$\mathrm{H}$ ispanic Americans are projected to become the largest ethnic minority group in the United States by $2005,{ }^{1}$ with two-thirds being of Mexican decent. ${ }^{2}$ Mexican Americans are a culturally and ethnically diverse group. However, little is known about how these variations relate to the mental heath of older Mexican Americans. The largest and most recent study is the Hispanic Established Populations for Epidemiologic Studies of the Elderly (H-EPESE), which reported an overall prevalence of depression (a score of 16 or more) on the Center for Epidemiologic Studies-Depression ${ }^{3}$ (CES-D) scale of 25.6\%. ${ }^{4}$ This is higher than other prevalence reports of depression in older non-Hispanic Caucasians and African Americans using the CES-D, which ranged from 9-16.9\%..$^{5-7}$ Three previous studies of older Mexican Americans have reported prevalence estimates of $13.2 \%, 20 \%$, and $30 \% .^{8-10}$ Thus, there are inconsistent estimates of the prevalence of depression among older Mexican Americans. In addition, little is known about how cultural differences in older Mexican Americans, as a group, affect depression.

Through U.S. westward expansion and more recently through immigration, acculturation from Mexican- to Anglo-oriented language and cultural practices has formed an important aspect of the social experience of many Mexican Americans. Changes in life style associated with acculturation have been related to increases in the risk of obesity, ${ }^{11}$ diabetes mellitus, ${ }^{12}$ and cardiovascular disease. ${ }^{13}$ 
Studies examining the association between acculturation and the risk of depression in Mexican Americans have utilized differing definitions and measures of acculturation and have had conflicting results. ${ }^{14}$ In comparison with U.S.-born Mexican Americans, Black et al. ${ }^{4}$ reported that immigrant women in H-EPESE were at significantly greater risk for depression, whereas being an immigrant male imparted a protective effect. Krause et al. ${ }^{15}$ examined English language use as a measure of acculturation and found that older Mexican Americans who used more English were at lower risk for depression. Results from the Hispanic Health and Nutrition Examination Survey reported opposite findings, in which less-acculturated, immigrant Mexican Americans were at a reduced risk of depression. However, that sample had a combined sample of younger and older adults. ${ }^{8}$ Therefore, disagreement remains in risk estimates of depression for acculturation and immigration subgroups of older Mexican Americans.

The purpose of this study was to estimate the prevalence and risk of depression in acculturation and immigration history subgroups of older Mexican Americans and determine which psychosocial, behavioral, and health factors are associated with depression. It was expected that the Mexican-oriented group, which may encounter more cultural and language barriers than bicultural and Anglo-oriented groups, would have a higher prevalence of depression. The results presented here are based on crosssectional analyses from the baseline data of the Sacramento Area Latino Study on Aging (SALSA), a population-based prospective study of the health of Latinos age 60 and older.

\section{METHODS}

\section{Participants}

Participants in this study were recruited as part of the SALSA. The Institutional Review Board of the University of California, Davis reviewed and approved SALSA. SALSA recruitment targeted older Latinos residing within the Census tracts of Sacramento, Yolo, Sutter, Solano, San Joaquin, and Placer Counties with proportional densities of Latinos greater than $10 \%$ based on updated 1990 U.S. Census information. ${ }^{16}$ The recruitment method was designed to enumerate all Latino households within these tracts. Participants were eligible for SALSA if (1) they selfidentified as being of Hispanic or Latino ancestry (parents or grandparents), (2) were over the age of 60, and (3) English, Spanish, or both were their primary languages. One thousand seven hundred and eighty-nine were recruited from March 1998 through June 1999, with an 82.2\% response rate. Bilingual/bicultural technicians conducted the survey interviews in participant's homes to obtain demographic, health, and functional status information. Survey instruments were translated from English to Spanish and then back-translated to English to ensure the accuracy of the translations. However, some instruments were already available in Spanish (e.g., Acculturation Ratings Scale for Mexican Americans-II) and had been field-tested. Bilingual participants selected the language with which they felt most comfortable for survey administration. Participants were interviewed and also had a brief clinical assessment for anthropometry, blood pressure, and a blood draw.

\section{Measures}

The CES-D was administered to all SALSA participants. The CES-D has been widely used in research as a measure of depressive symptoms in geriatric populations ${ }^{17-19}$ and older Latinos, ${ }^{20,21}$ and it consists of 20 four-point Likerttype questions with a total score range of 0 to 60 . The CES-D was dichotomized using a standard cutoff score of 16 or greater to classify individuals with elevated depressive symptoms. ${ }^{3}$ ARSMA-II was modified for use with older Latinos to measure their level of acculturation. The Geriatric Acculturation Ratings Scale for Mexican Americans (G-ARSMA) consisted of 19 items and assessed English and Spanish language and media use, childhood and current friendships, contact with Latin America, and dietary practices. The scoring procedures were similar to those recommended by Cuellar et al. ${ }^{22}$ The total score was then categorized into tertiles with the lowest tertile representing the least acculturated (Mexican-oriented), the middle tertile representing bicultural participants, and the top tertile representing Anglo-oriented participants.

Sociodemographic variables included participants' age, education, gender, marital status, place of birth, monthly income level, and current living arrangements. Health behaviors that could potentially be associated with depressive symptoms included physical activity, smoking status (never smoked, former and current smoker), and alcohol use. A physical activity score was assessed by asking participants the number of hours per week they engaged in activities such as walking, standing, gardening, and light and heavy household chores. ${ }^{23,24}$ This measure was divided into tertiles of physical activity ranging from less to highly active. Alcohol use groups were defined as nondrinkers, moderate drinkers ( 1 to 3 drinks per sitting), and heavy drinkers (4 or more). Medical history and functional status information was based on participants' self-report. The Katz activities of daily living (ADL) scale ${ }^{25}$ was used to measure grooming, toileting, and other basic activities. Consistent with approach used by H-EPESE, individuals requiring assistance for any of these basics activities were grouped as impaired. ${ }^{4}$ Arthritis was defined by the number of areas of affected joints (hands, shoulders, and knees) and ranged from 0 to 3 . Self-reported medical diagnoses of diabetes mellitus, hypertension, heart disease, stroke, and cancer were categorized according to the presence or absence of the disorder.

\section{Statistical Approach}

The sum of CES-D scores was positively skewed, leptokurtotic, and significantly non-normally distributed, (Kolmogorov-Smirnov $\mathrm{Z}=7.04, P<.001)$, with the majority of scores $(74.6 \%)$ lower than the 16 -point cutoff. The prevalence of depression was calculated for age groups by gender, categorical education, marital status, birthplace, acculturation category, and income. Logistic regression analyses were used to examine the association between the CES-D scores and acculturation, immigration history, psychosocial, behavioral, and health variables. For categorical measures, indicator variables were used. For each risk factor, a univariate model was tested first. Variables that were statistically significant in the univariate analyses $(P \leq .05)$ were retained in subsequent multivariate models. To determine the 
relationship between birthplace, acculturation, and depression, birthplace and acculturation were simultaneously entered into a logistic regression model. A series of multivariate logistic regression models were used to examine the association between psychosocial, behavioral, and health predictor variables and depression. All models included age and gender. Model 1 assessed the association between acculturation and depression. Models 2, 3, and 4 examined the independent and simultaneous effects of education and monthly income on the relationship between acculturation and depression. Marital status, living arrangements, physical activity, and alcohol consumption were statistically significant in the univariate models and were included in Model 5 to assess them as confounders of the association between acculturation and depression. Finally, chronic health problems (ADL impairment, arthritis, diabetes mellitus, hypertension, heart disease, stroke, and cancer) were included in Model 6 to assess them as confounders of the association between acculturation and depression.

\section{RESULTS}

Demographic characteristics of SALSA participants are presented in Table 1 . Of the 1,789 SALSA participants, 1,663 had complete data available for this study. The number of depressed cases in the complete and missing data groups were not significantly different $\left(\chi^{2}=5.22, P=\right.$ .16). The SALSA sample was equally distributed between U.S.-born participants $(48.9 \%)$ and immigrants $(51.1 \%)$; the majority of the immigrant sample was born in Mexico $(89.0 \%)$.

The results of the univariate logistic regression analyses are shown in Table 2 for the overall sample and for U.S.-born and immigrant groups. Older age $(80+)$, lower levels of education, female gender, unmarried status, and low monthly income were associated with significantly increased risks of depression. Bicultural and Mexican-oriented participants had a higher risk of depression than Anglo-oriented participants. Among U.S.-born participants, the risk of depression for either bicultural or Mexican-oriented was notably higher than for Anglo-oriented participants. Among immigrants, Mexican-oriented participants were more depressed but bicultural participants were not significantly more depressed compared with Anglo-oriented participants.

Acculturation and immigration were strongly associated. Table 3 presents acculturation by birthplace. The majority of SALSA participants born elsewhere were from Central America (67\%), followed by South America (20\%), with the remainder being from Caribbean countries. There

Table 1. Sacramento Area Latino Study on Aging (SALSA) Social, Cultural, and Health-Related Characteristics and their Relationships with the Prevalence of Depression (Center for Epidemiologic Studies-Depression Score ${ }^{3} \geq 16$ )

\begin{tabular}{|c|c|c|c|c|c|c|c|}
\hline & \multicolumn{3}{|c|}{ Total } & \multicolumn{2}{|c|}{ U.S.-Born } & \multicolumn{2}{|r|}{ Immigrant } \\
\hline & $\mathrm{N}$ & $\%$ & Prevalence (\%) & $\mathrm{N}$ & Prevalence (\%) & $\mathrm{N}$ & Prevalence (\%) \\
\hline Total & 1,663 & 100.0 & 25.4 & & & & \\
\hline \multicolumn{8}{|l|}{ Age groups } \\
\hline $60-69$ & 835 & 50.2 & 23.5 & 425 & 18.4 & 410 & 28.8 \\
\hline $70-79$ & 662 & 39.8 & 25.8 & 348 & 21.8 & 314 & 30.3 \\
\hline $80+$ & 166 & 10.0 & 33.7 & 58 & 27.6 & 108 & 37.0 \\
\hline \multicolumn{8}{|l|}{ Education years } \\
\hline $12+$ & 499 & 30.0 & 13.4 & 382 & 11.5 & 117 & 19.7 \\
\hline $4-11$ & 644 & 38.7 & 25.3 & 331 & 25.1 & 313 & 25.6 \\
\hline $0-3$ & 520 & 31.3 & 37.1 & 118 & 36.4 & 402 & 37.3 \\
\hline \multicolumn{8}{|l|}{ Gender } \\
\hline Male & 695 & 41.8 & 16.3 & 360 & 13.1 & 335 & 19.7 \\
\hline Female & 968 & 58.2 & 32.0 & 471 & 26.1 & 497 & 37.6 \\
\hline \multicolumn{8}{|l|}{ Marital status } \\
\hline Married/cohabitating & 989 & 59.5 & 21.9 & 492 & 17.3 & 497 & 26.6 \\
\hline Single/divorced/separated & 273 & 16.4 & 28.2 & 156 & 26.3 & 117 & 30.8 \\
\hline Widowed & 401 & 24.1 & 32.2 & 183 & 24.0 & 218 & 39.0 \\
\hline \multicolumn{8}{|l|}{ Birthplace } \\
\hline U.S.-born & 831 & 50.0 & 20.5 & & & & \\
\hline Immigrant & 832 & 50.0 & 30.4 & & & & \\
\hline \multicolumn{8}{|l|}{ Acculturation groups } \\
\hline Anglo-oriented & 553 & 33.3 & 16.1 & 492 & 15.7 & 61 & 19.7 \\
\hline Bicultural & 562 & 33.8 & 24.2 & 281 & 24.9 & 281 & 23.5 \\
\hline Mexican-oriented & 548 & 33.0 & 36.1 & 58 & 39.7 & 490 & 35.7 \\
\hline \multicolumn{8}{|l|}{ Monthly income } \\
\hline$\$ 2,000$ & 396 & 23.8 & 10.4 & 307 & 8.8 & 89 & 15.7 \\
\hline$\$ 1,000-1,999$ & 534 & 32.1 & 22.3 & 283 & 19.8 & 251 & 25.1 \\
\hline$<\$ 1,000$ & 733 & 44.1 & 35.9 & 241 & 36.1 & 492 & 35.8 \\
\hline
\end{tabular}


Table 2. Relation to Risk Factors to Depression (Center for Epidemiologic Studies-Depression Score $\geq 16$ ) for the Total Sample, U.S.-Born and Immigrant Participants of the Sacramento Area Latino Study on Aging $(N=1,663)$ from Univariate Logistic Regressions

\begin{tabular}{|c|c|c|c|c|c|c|}
\hline & \multicolumn{2}{|c|}{ Total Sample } & \multicolumn{2}{|c|}{ U.S.-Born } & \multicolumn{2}{|c|}{ Immigrant } \\
\hline & OR & $95 \% \mathrm{Cl}$ & OR & $95 \% \mathrm{Cl}$ & OR & $95 \% \mathrm{Cl}$ \\
\hline \multicolumn{7}{|l|}{ Age groups } \\
\hline $60-69$ & 1.00 & & 1.00 & & 1.00 & \\
\hline $70-79$ & 1.14 & $0.90-1.44$ & 1.24 & $0.87-1.77$ & 1.07 & $0.78-1.48$ \\
\hline $80+$ & 1.66 & $1.16-2.38$ & 1.69 & $0.91-3.17$ & 1.46 & $0.93-2.27$ \\
\hline \multicolumn{7}{|l|}{ Education years } \\
\hline $12+$ & 1.00 & & 1.00 & & 1.00 & \\
\hline $4-11$ & 2.18 & $1.60-2.98$ & 2.57 & $1.72-3.84$ & 1.40 & $0.83-2.36$ \\
\hline $0-3$ & 3.80 & $2.78-5.20$ & 4.40 & $2.70-7.18$ & 2.43 & $1.48-4.01$ \\
\hline Gender: female & 2.43 & $1.90-3.09$ & 2.35 & $1.63-3.40$ & 2.46 & $1.78-3.40$ \\
\hline \multicolumn{7}{|l|}{ Marital status } \\
\hline Married/cohabitating & 1.00 & & 1.00 & & 1.00 & \\
\hline Single/divorced/separated & 1.40 & $1.03-1.89$ & 1.71 & $1.11-2.61$ & 1.23 & $0.79-1.91$ \\
\hline Widowed & 1.69 & $1.30-2.18$ & 1.52 & $1.00-2.29$ & 1.77 & $1.26-2.48$ \\
\hline \multicolumn{7}{|l|}{ Acculturation groups } \\
\hline Anglo-oriented & 1.00 & & 1.00 & & 1.00 & \\
\hline Bicultural & 1.66 & $1.24-2.24$ & 1.79 & $1.24-2.57$ & 1.25 & $0.63-2.50$ \\
\hline Mexican-oriented & 2.95 & $2.22-3.93$ & 3.54 & $1.98-6.32$ & 2.27 & $1.18-4.38$ \\
\hline \multicolumn{7}{|l|}{ Monthly income } \\
\hline$\geq \$ 2,000$ & 1.00 & & 1.00 & & 1.00 & \\
\hline$\$ 1,000-1,999$ & 2.48 & $1.69-3.64$ & 2.56 & $1.56-4.18$ & 1.79 & $0.95-3.40$ \\
\hline$<\$ 1,000$ & 4.85 & $3.39-6.92$ & 5.86 & $3.65-9.42$ & 2.98 & $1.64-5.43$ \\
\hline
\end{tabular}

$\mathrm{OR}=$ odds ratio $\mathrm{CI}=$ confidence interval

were clear differences in acculturation, with most of the U.S.-born participants Anglo-oriented and those born in Mexico and elsewhere Mexican-oriented and bicultural, respectively.

\section{Multivariate Models}

A logistic regression model, including age and gender was used to assess the effects of covariate adjustment on the association between acculturation and birthplace. The association between birthplace and depression was no longer statistically significant (OR (U.S./immigrant) $=0.95(0.70$ 1.28)) and the association between acculturation and depression was not affected by this adjustment. Because the association between birthplace and depression was almost entirely explained by acculturation, birthplace was elimi-

Table 3. Association between Birthplace and Acculturation for Participants of the Sacramento Area Latino Study on Aging $(\mathrm{N}=1,663)$

\begin{tabular}{|c|c|c|c|c|c|c|}
\hline \multirow{2}{*}{$\begin{array}{c}\text { Acculturation } \\
\text { Groups }\end{array}$} & \multicolumn{2}{|c|}{ U.S.-Born } & \multicolumn{2}{|c|}{$\begin{array}{c}\text { Mexico } \\
\text { Born }\end{array}$} & \multicolumn{2}{|c|}{$\begin{array}{c}\text { Born } \\
\text { Elsewhere }\end{array}$} \\
\hline & $\mathrm{N}$ & $\%$ & $\mathrm{~N}$ & $\%$ & $\mathrm{~N}$ & $\%$ \\
\hline Anglo-oriented & 492 & 59.21 & 53 & 7.17 & 8 & 8.60 \\
\hline Bicultural & 281 & 33.81 & 238 & 32.21 & 43 & 46.24 \\
\hline Mexican-oriented & 58 & 6.98 & 448 & 60.62 & 42 & 45.16 \\
\hline
\end{tabular}

nated from the remaining multivariate models. Table 4 presents a series of six age- and gender-adjusted multivariate logistic regression models. In Model 1, bicultural and Mexican-oriented groups were at significantly higher risk of depression than the Anglo-oriented group. When education level was introduced into Model 2, the risk of depression was significantly elevated only for the Mexican-oriented group. When monthly income was added to Model 3, the risk of depression was reduced for the bicultural and Mexican-oriented groups but remained elevated. However, when education and monthly income were simultaneously entered into Model 4, the risk of depression fell below significance $(P<.05)$ for all the acculturation groups. Adjustment for psychosocial and behavioral risk factors had little effect on the relationship between acculturation and depression in Model 5. However, when chronic health problems were introduced into Model 6, the risk of depression for the Mexican-oriented group was statistically significant but not substantially increased $(10 \%)$ above Model 5 .

\section{DISCUSSION}

The prevalence of depression for older Mexican Americans in SALSA was nearly identical to what was recently reported from H-EPESE, ${ }^{4}$ which is higher than reports for non-Hispanic Caucasians ${ }^{5}$ and African Americans. ${ }^{6,7}$ Thus, there is convergence from two large population-based studies indicating that more than one in four older Mexican Americans are experiencing major psychological distress. Important questions remain about interpreting these findings. Because the CES-D is an index of clinical depres- 
Table 4. Relationship of Risk Factors to Depression (Center for Epidemiologic Studies-Depression Score $\geq 16$ ) for Participants of the Sacramento Area Latino Study on Aging $(\mathrm{N}=1,663)$ from Multivariate Logistic Regression Models

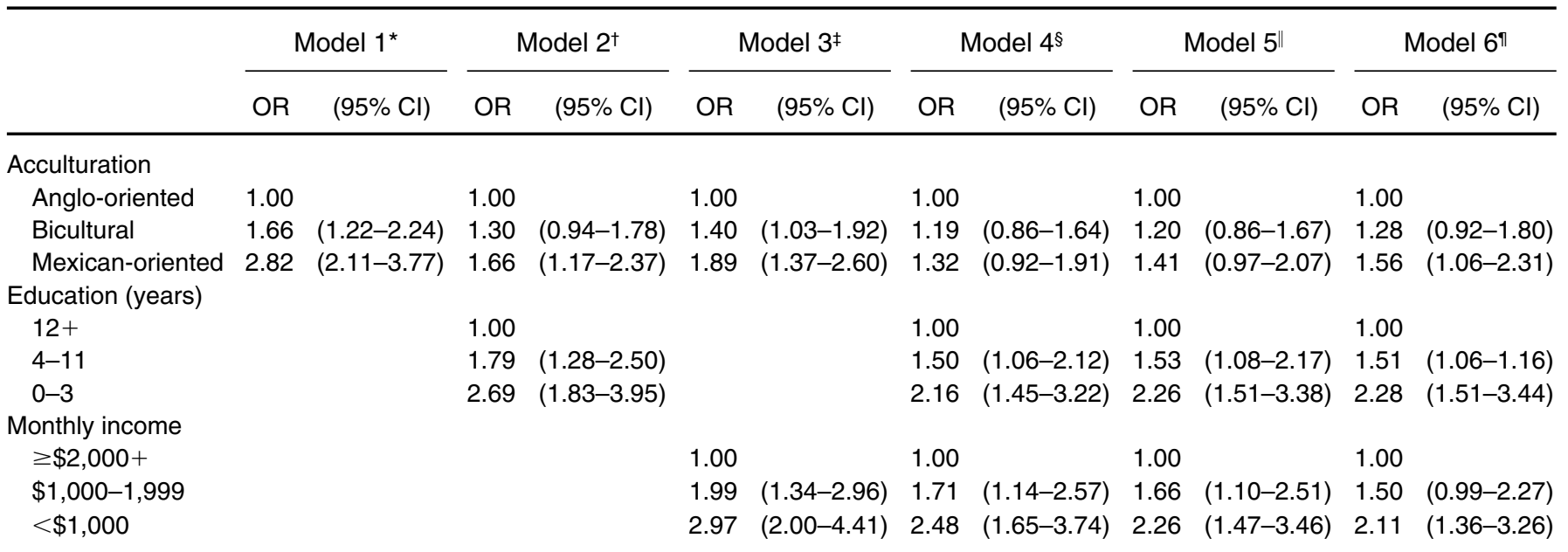

Note: All models are age- and gender-adjusted.

*Acculturation.

${ }^{\dagger}$ Acculturation and education in years.

${ }^{\ddagger}$ Acculturation and monthly income.

$\S$ Acculturation, education in years, and monthly income.

Acculturation, education in years, monthly income, and psychosocial and behavior (marital status, living arrangements, physical activity, and alcohol consumption).

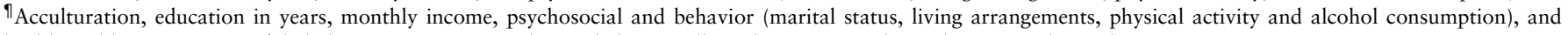
health problems (activities of daily living impairment, arthritis, diabetes mellitus, hypertension, heart disease, stroke, and cancer).

$\mathrm{OR}=$ odds ratio; $\mathrm{CI}=$ confidence interval.

sion, ${ }^{26}$ these findings suggest that older Mexican Americans may be at higher risk for clinical depression. Alternatively, the sensitivity and specificity characteristics of the standard cutoff of the CES-D among Mexican Americans may differ from non-Hispanic Caucasians. In the absence of studies validating the CES-D against clinical measures of depression in this same population, it is difficult to extrapolate from these results to the prevalence of clinical depression in this population.

An attempt to validate the CES-D against the Diagnostic Interview Schedule (DIS), which generates Diagnostic and Statistical Manual III (DSM-III) diagnoses, with English- and Spanish-speaking Mexican-American and nonHispanic Caucasian younger adult psychiatric patients, found general agreement between the CES-D and a DSMIII diagnosis of Major Depressive Disorder across all three groups. They also reported CES-D and DIS agreement of a DSM-III diagnosis of Generalized Anxiety Disorder for non-Hispanics and English-speaking Hispanics, but not for Spanish-speaking Mexican Americans. ${ }^{27}$ Although that validation study was limited to younger adults, the results support the contention that the CES-D measures significant psychological distress in the form of depression in English- and Spanish-speaking Mexican Americans. If that is the case, and given the high prevalence of depression that has been reported by H-EPESE and now SALSA, public health efforts should be made to educate health professionals on the high risk of depression in older Mexican Americans and to promote screening for depression in older Mexican Americans. Given the public health importance of clinical depression, these questions are critical areas for further investigation.

\section{Associations of Acculturation, Immigration, and Depression}

A major finding of this study, and to our knowledge the first report of this finding, is that the prevalence and risk of depression was higher for older immigrants than U.S.born Mexican Americans. This contrasts with previous reports of a higher prevalence of depression among younger U.S.-born Mexican Americans than among Mexican immigrants. ${ }^{8,28,29}$ In light of the interest in understanding the impact of immigration on psychopathology among younger Mexican Americans, it is surprising that there is so little information available on this relationship in older adults. Using data from the Los Angeles Epidemiologic Catchment Area Project, Sorenson et al. ${ }^{30}$ reported that U.S.-born Mexican Americans had an earlier age of onset of depression than immigrants. One possible explanation is that because immigration requires substantial effort it would present a greater obstacle for depressed younger adults. As a result, delays in age of onset for immigrants may account for the differences in prevalences between young and older Mexican Americans. There may also be cohort effects influencing the differences in prevalence of depression, such as fewer educational and economic opportunities compared with younger adults. No studies, to our knowledge, have addressed the crossover in prevalence based on birthplace; further studies are needed to understand this phenomenon.

Another major finding of this study was that, for older Mexican Americans, the association between birthplace and depression was entirely explained by acculturation. This is the first study, to our knowledge, that has examined the prevalence and risk of depression in older 
Mexican Americans that included bicultural Mexican Americans. This contrasts with earlier approaches that view acculturation as an "either/or" process that is based on the faulty assumption that Mexican culture must be abandoned to adapt to the dominant Anglo culture. ${ }^{22}$ Based on the findings of this study, it is recommended that future studies of ethnic minorities with large immigrant populations should assess the association between acculturation and immigration history before deciding to include these two variables in one analysis. Failure to do so increases the chances of missing important associations between acculturation, immigration, and the outcome variable under investigation.

It is very likely that older U.S.-born Mexican Americans are more acculturated than immigrants. However, acculturation is not a monolithic construct but is dynamic and situationally dependent. Therefore, acculturated and bicultural individuals may have important language and cultural understandings of the dominant culture that permits them greater access to educational and job opportunities, which in turn may increase their socioeconomic standing. In addition, language and cultural fluency with U.S. culture could increase access to social and medical resources. Conversely, less-acculturated Mexican Americans may lack the language and cultural fluency needed to effectively negotiate educational and work-related demands that would provide opportunities for advancement, which in turn could limit financial earnings and result in hardship. Language barriers in particular have been shown to promote social isolation ${ }^{31}$ and limit access to health care. ${ }^{32}$ Under these conditions, perceived self-efficacy would be diminished and psychological distress could result. Prospective research is needed to evaluate this model and to understand the high prevalence of depression among older Mexican Americans.

Based on the convergent findings from H-EPESE and SALSA, clinicians treating older Mexican Americans are advised to routinely assess for depression, particularly in patients who are less acculturated and whose primary language is Spanish. For clinicians with limited Spanish language skills, adequately trained interpreters are essential to ensure that language and cultural barriers do not impede accurate assessments, appropriate referrals and treatments. ${ }^{33}$

\section{ACKNOWLEDGMENTS}

The authors would like to acknowledge the efforts of Teresa Ortiz, RN, Esther L. Rivera, the staff, and especially the generous participants of the Sacramento Area Latino Study on Aging.

\section{REFERENCES}

1. U.S. Census Bureau. National Population Projections. Washington, DC: U.S. Government Printing Office, 2000.

2. U.S. Census Bureau. The Hispanic Population of the United States. Washington, DC: U.S. Government Printing Office, 2000.

3. Radloff LS. The CES-D scale: A self-report depression scale for research in the general population. Appl Psych Meas 1977;1:385-401.

4. Black SA, Markides KS, Miller TQ. Correlates of depressive symptomatology among older community-dwelling Mexican Americans: The Hispanic EPESE. J Gerontology B Psych Sci Soc Sci 1998;53B(4):S198-S208.

5. Berkman LF, Berkman CS, Kasl S et al. Depressive symptoms in relation to physical health and functioning in the elderly. Am J Epidemiol 1986;124: 372-388.

6. Blazer DG, Landerman LR, Hays JC et al. Symptoms of depression among community-dwelling elderly African-American and white older adults. Psychol Med 1998;28:1311-1320.

7. Murrell SA, Himmelfarb S, Wright K. Prevalence of depression and its correlates in older adults. Am J Epidemiol 1983;117:173-185.

8. Moscicki EK, Locke BZ, Rae DS et al. Depressive symptoms among Mexican Americans: The Hispanic Health and Nutrition Examination Survey. Am J Epidemiol 1989;130:348-360.

9. Mendes de Leon CF, Markides KS. Depressive symptoms among Mexican Americans: A three-generation study. Am J Epidemiol 1988;127:150-160.

10. Vernon SW, Roberts RE. Prevalence of treated and untreated psychiatric disorders in three ethnic groups. Soc Sci Med 1982;16:1575-82.

11. Sundquist J, Winkleby M. Country of birth, acculturation status and abdominal obesity in a national sample of Mexican-American women and men. Int J Epidemiol 2000;29:470-477.

12. Hazuda HP, Haffner SM, Stern MP et al. Effects of acculturation and socioeconomic status on obesity and diabetes in Mexican Americans. The San Antonio Heart Study. Am J Epidemiol 1988;128:1289-1301.

13. Sundquist J, Winkleby MA. Cardiovascular risk factors in Mexican American adults: A transcultural analysis of NHANES III, 1988-1994. Am J Public Health 1999;89:723-730.

14. Rogler LH, Cortes DE, Malgady RG. Acculturation and mental health status among Hispanics: Convergence and new directions for research. Am Psychol 1991;46:585-597.

15. Krause N, Goldenhar LM. Acculturation and psychological distress in three groups of elderly Hispanics. J Gerontol 1992;47:S279-88.

16. U.S. Census Bureau. Current Population Series. Washington, DC: U.S. Government Printing Office, 1996.

17. Bassuk SS, Berkman LF, Wypij D. Depressive symptomatology and incident cognitive decline in an elderly community sample. Arch Gen Psychiatry 1998; 55:1073-1081.

18. Lyness JM, Noel TK, Cox C et al. Screening for depression in elderly primary care patients. A comparison of the Center for Epidemiologic Studies-Depression Scale and the Geriatric Depression Scale. Arch Intern Med 1997;157: 449-454.

19. Gatz M, Johansson B, Pedersen N et al. A cross-national self-report measure of depressive symptomatology. Int Psychogeriatr 1993;5:147-156.

20. Black SA, Goodwin JS, Markides KS. The association between chronic diseases and depressive symptomatology in older Mexican Americans. J Gerontol A Biol Sci Med Sci 1998;53A(3):M188-94.

21. Grigsby J, Kaye K, Baxter J et al. Executive cognitive abilities and functional status among community-dwelling older persons in the San Luis Valley Health and Aging Study. J Am Geriatr Soc 1998;46:590-596.

22. Cuellar I, Arnold B, Maldonado R. Acculturation Rating Scale for Mexican Americans-II: A revision of the original ARSMA Scale. Hispanic J Behav Sci 1995;17(3):275-304.

23. Fried LP, Borhani NO, Enright P et al. The Cardiovascular Health Study: Design and rationale. Ann Epidemiol 1991;1:263-276.

24. Taylor HL, Jacobs DR Jr, Schucker B et al. A questionnaire for the assessment of leisure time physical activities. J Chron Dis 1978;31:741-755.

25. Katz SC, Ford AB, Moskowitz RW et al. Studies of illness in the aged: The index of ADL, a standardized measure of biological and psychosocial function. JAMA 1963;185:914-919.

26. Radloff LS, Teri L. Use of the Center for Epidemiological Studies-Depression Scale with older adults. Clin Gerontol 1986;5:119-36.

27. Roberts RE, Rhoades HM, Vernon SW. Using the CES-D scale to screen for depression and anxiety: Effects of language and ethnic status. Psychiatry Res 1990;31:69-83.

28. Golding JM, Burnam MA. Immigration, stress, and depressive symptoms in a Mexican-American community. J Nerv Ment Dis 1990;178:161-71.

29. Vega WA, Kolody B, Aguilar-Gaxiola S et al. Lifetime prevalence of DSMIII-R psychiatric disorders among urban and rural Mexican Americans in California. Arch Gen Psychiatry 1998;55:771-78.

30. Sorenson SB, Rutter CM, Aneshensel CS. Depression in the community: An investigation into age of onset. J Consult Clin Psychol 1991;59:541-46.

31. O'Donnell RM. Functional disability among the Puerto Rican elderly. J Aging Health 1989;1:244-6.

32. Woodward AM, Dwinell AD, Arons BS. Barriers to mental health care for Hispanic Americans: A literature review and discussion. J Ment Health Admin 1992;19:224-236.

33. American Psychological Association. Guidelines for Providers of Psychological Services to Ethnic, Linguistic, and Culturally Diverse Populations [Online]. Available: http://www.apa.org/pi/oema/guide.html, 1990. 